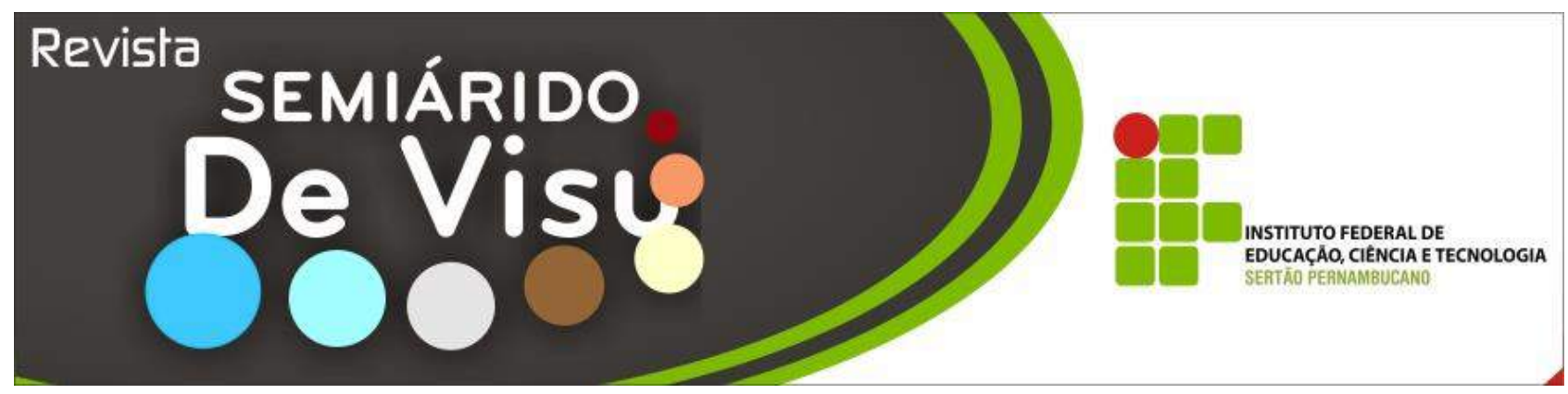

\title{
Ensino-aprendizagem de física via mínimos quadrados com auxílio de planilha eletrônica
}

\author{
Francisco Joceildo da Silva ${ }^{1}$, Eriverton da Silva Rodrigues ${ }^{2}$, Alisson dos Anjos Gomes ${ }^{3}$, Cicero Jailton de Morais \\ Souza $^{4}$ \\ 1,2,3,4 Instituto Federal de Educação, Ciência e Tecnologia Sertão Pernambucano - Campus Salgueiro. Rodovia Luiz Gonzaga (BR

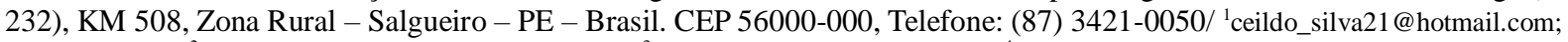 \\ 2eriverton.rodrigues@ifsertao-pe.edu.br; ${ }^{3}$ alissongomespe@ outlook.com; ${ }^{4}$ cicero.jailton@ifsertao-pe.edu.br
}

RESUMO: A precisa realização desse projeto buscou promover a utilização do Método dos Mínimos Quadrados utilizando a planilha eletrônica como ferramenta computacional no ensino de Física Experimental para alunos do ensino médio. Neste sentido, se trata de uma forma de aproximar resultados de medidas experimentais reduzindo ao máximo as flutuações que aparecem dentro desse conjunto, esses desvios precisam ser justificados ao máximo para que se possa obter um valor experimental com uma precisão considerável. Para a aplicação desse recurso foi utilizado o experimento de resistividade elétrica com a segunda lei de Ohm, este que consiste em medir a resistência de diferentes tipos de material variando sua área e seu comprimento.

Palavras-chaves: Lei de Ohm, Resistividade, Física Experimental.

\section{Physical education via least-squares with learning worksheet aid electronics}

\begin{abstract}
The precise realization of this project sought to promote the use of the least squares method using the spreadsheet as a computational tool in experimental physics teaching for high school students. In this sense, it is a way to bring results of experimental measures while minimizing the fluctuations that appear within that set, these deviations must be justified to the maximum so that you can obtain an experimental value with considerable precision. To implement this feature was used resistivity experiment with the second Ohm's law, this consisting in measuring the resistance of different types of material varying its length and its area.
\end{abstract}

Keywords: Ohm's Law, Resistivity, Experimental Physics. 
(SILVA et al., 2016)

\section{Introdução}

$\mathrm{N}$ os últimos anos, muito se tem falado a respeito do alto grau de dificuldade dos alunos na aprendizagem de Física, do alto índice de rejeição e da desvinculação da Física do cotidiano, e da aplicação do que é ensinado na Física do Ensino Médio ao mesmo. A respeito disso, Solbes e Vilches; Jiménez e Otero apud Carvalho e Gil-Pérez (2000) afirmam que:

\begin{abstract}
(...) a ideia de que fazer Ciência é pouco menos que trancar-se em uma torre de marfim - "no mundo dos livros" ou coisa parecida distanciado da realidade, constitui uma imagem tópica bastante difundida e com qual nosso ensino lamentavelmente contribui, reduzindo a Ciência à transmissão de conteúdos conceituais e, se muito, treinamento em alguma destreza, deixando de lado os aspectos históricos, sociais etc. que marcam o desenvolvimento científico. (p.24).
\end{abstract}

O ensino de Física é tratado como mecanicista, que admite um conhecimento sem significado para o aluno (CARVALHO e PÉREZ, 2000). O desenvolvimento de uma atividade prática inovadora para o ensino de Física no ensino médio é de suma importância para reverter essa visão. Segundo Brasil (2002), é importante trabalhar o conhecimento de física contextualizado, para favorecer aos alunos uma formação de um cidadão participante e atuante no mundo contemporâneo.

A atividade experimental usando o método dos mínimos quadrados que procura aperfeiçoar o ajuste dos dados obtidos no experimento, arranjando-os em um valor ótimo que se aproxima do valor teórico esperado pelos alunos, para avaliar o quanto a atividade interfere na construção do conhecimento.

Um dos objetivos relevantes desta pesquisa foi testar a eficácia do método dos mínimos quadrados na problematização de medidas experimentais sobre a lei de ohm e favorecer aos alunos do nível médio, por meio de uma atividade experimental, que faz uso da informática através do Método dos Mínimos Quadrados no Excel, maior conhecimento do estudo do movimento uniforme e suas características e aplicações.

\section{Material e métodos}

A precisa realização deste estudo é baseada em aulas práticas de Física Experimental III, com a participação dos estudantes do ensino médio integrado do Instituto Federal do Sertão Pernambucano Campus Salgueiro (IF Sertão PE - Campus Salgueiro). Essas aulas foram ministradas no segundo semestre de 2015 para uma turma do $3^{\circ}$ ano do ensino médio integrado de informática estas que tem como objetivo mostrar aos alunos a teoria envolvida na pesquisa que são a resistividade elétrica dos materiais e o método dos mínimos quadrados através de recursos computacionais, pois como retrata Mees (2002), o uso do computador como ferramenta pode tornar o ensino de Física mais significativo. As aulas para pesquisa de campo ocorreram nas dependências do Laboratório de Física do IF Sertão PE - Campus Salgueiro.

Após a pesquisa documental e bibliográfica, na qual também tivemos preocupação com os conceitos históricos envolvidos, foi feita a aplicação de um questionário para termos ideia dos conhecimentos prévios dos alunos sobre os temas propostos na pesquisa e, posteriormente, foi feita a montagem e a realização dos experimentos, construção de trabalhos relacionados a pesquisa e preparação do relatório parcial. Daí, começamos a parte prática, na qual os materiais e recursos utilizados vão se transformando no nosso aparato experimental (Figura 1). Durante esse processo de montagem foi sempre posto em questão a possibilidade de utilizar outros tipos de materiais, que produzam o mesmo efeito e resposta para o experimento.

Para a realização do experimento aqui relatado foi utilizado um fio de Nicromo (liga metálica) com um diâmetro de $0,057 \mathrm{~cm}$; suporte para fixar e tencionar o fio; trena e multímetro. É importante salientar que os materiais utilizados para confecção do aparato experimental são de simples aquisição todos 
(SILVA et al., 2016)

disponíveis no laboratório de Física do IF Sertão Campus Salgueiro, exceto o fio de Nicromo, este que pode ser facilmente encontrado no comércio a um baixo custo. Também vale mencionar que se pode utilizar fios de outros materiais para a realização dessa atividade.

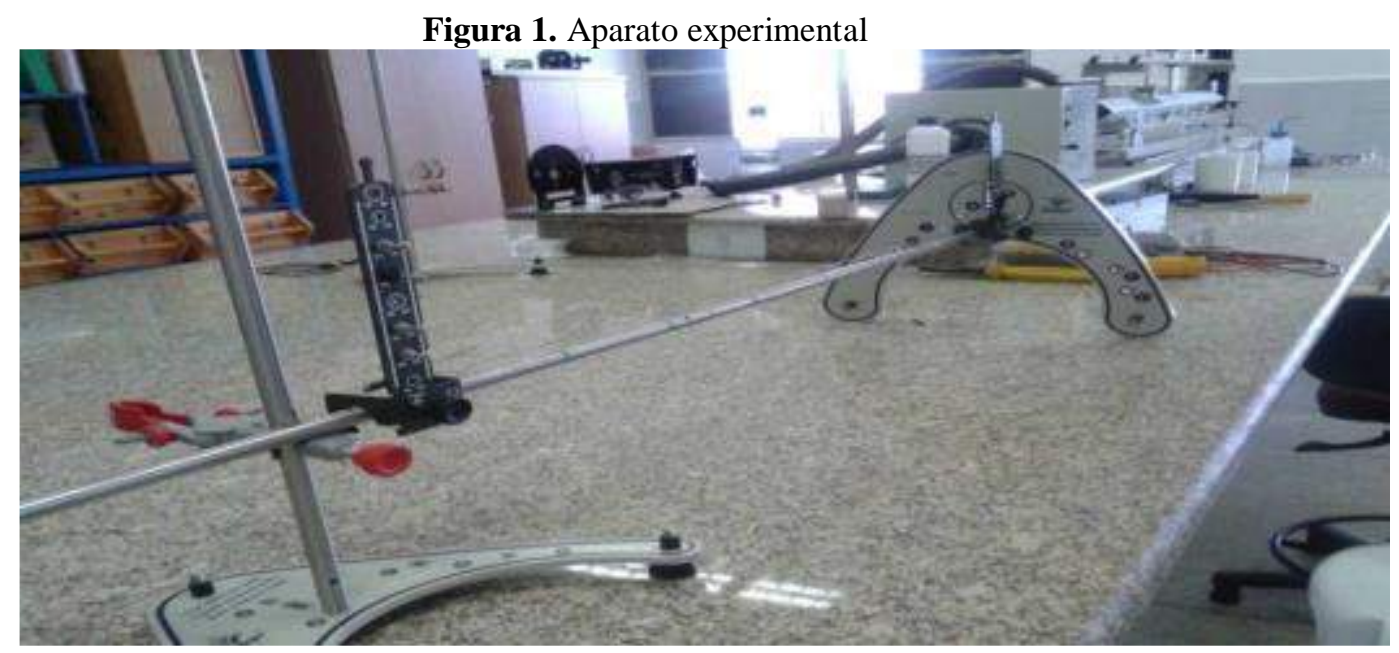

Fonte: Pesquisa direta.

Em seguida, com o multímetro, foram feitas cinco medidas de resistência elétrica no fio sempre variando o comprimento (Figura 2) de dez em dez centímetros.

Figura 2. Medições com o multímetro.

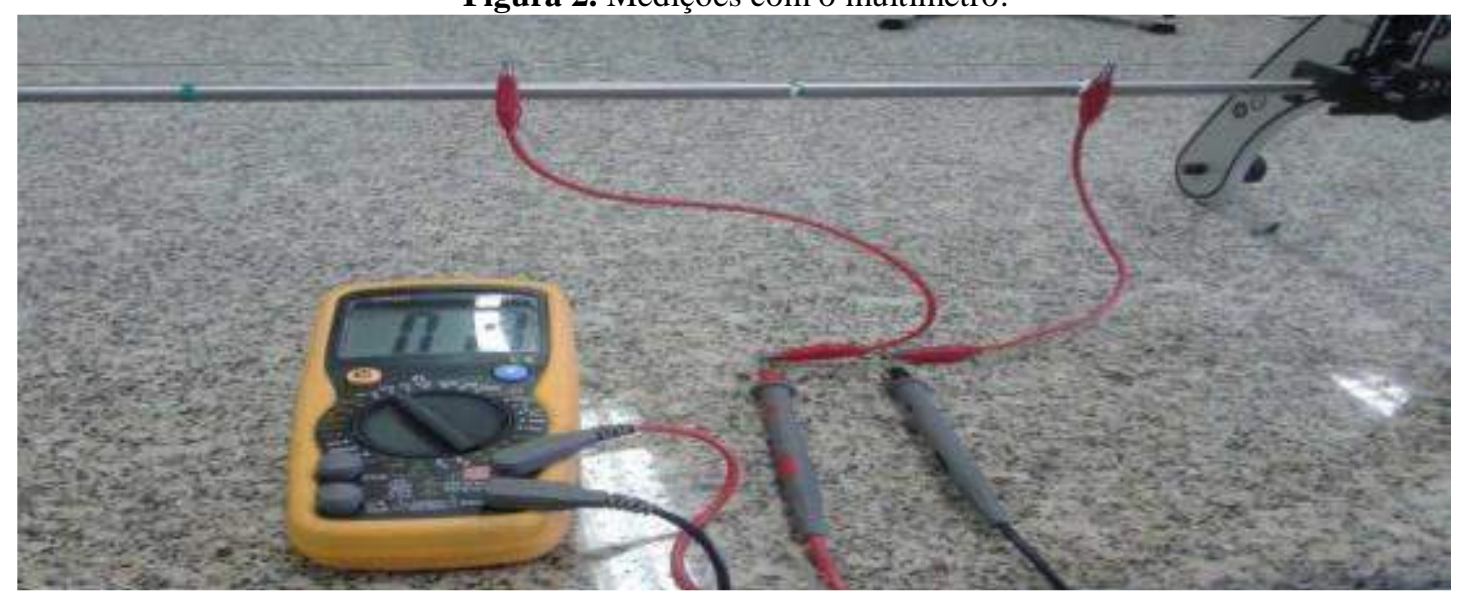

Fonte: Pesquisa direta.

\section{Resultados e discussão}

A pesquisa documental apresentou um aspecto peculiar em relação a utilização da planilha eletrônica no contexto educacional, de forma que ela já é bem conhecida e utilizada pelos docentes, mas somente são explorados seus recursos básicos, como por exemplo, fazer listas de frequência, elaborar tabelas, gráficos, etc. Segundo Betz e Ribeiro-Teixeira (2012), provavelmente um bom número de professores já faz uso desse recurso para tabelar resultados de avaliações, calcular médias e etc. Ainda seguindo esta linha, podemos observar conforme Morgado (2003), que as planilhas se constituem num meio dinâmico, pois possibilitam a manipulação direta sobre as representações matemáticas que se apresentam na tela do computador. Além disso, a introdução de recursos computacionais dentro do contexto ensino-aprendizado é citada em diversos trabalhos científicos e se mostra algo que vem crescendo, de forma que, se busca adequar o ensino nas escolas às novas tecnologias 
(SILVA et al., 2016)

desenvolvidas e fazer dessas tecnologias um veículo comunicador entre o discente e as matérias estudadas em sala de aula.

Os resultados obtidos nas medições foram aplicados na planilha do Excel de forma que, para cada valor $\mathrm{x}$ das resistências, existe um comprimento y associado, como se observa na Figura 3.

Figura 3. Tabela dos valores aplicados na planilha do Excel.

\begin{tabular}{|r|r|}
\hline $\mathbf{X}$ & \multicolumn{1}{|c|}{$\mathbf{Y}$} \\
\hline 1,06 & 0,1 \\
\hline 1,7 & 0,2 \\
\hline 2,2 & 0,3 \\
\hline 2,6 & 0,4 \\
\hline 3,13 & 0,5 \\
\hline & \\
\hline & \\
\hline & \\
\hline & \\
\hline & \\
\hline
\end{tabular}

Fonte: Planilha do Excel

Após a inserção dos valores das da planilha eletrônica calculamos os seguintes resistências e dos comprimentos, com o auxílio valores (Figura 4).

Figura 4. Cálculos do método dos mínimos quadrados referentes à resistência e ao comprimento do fio.

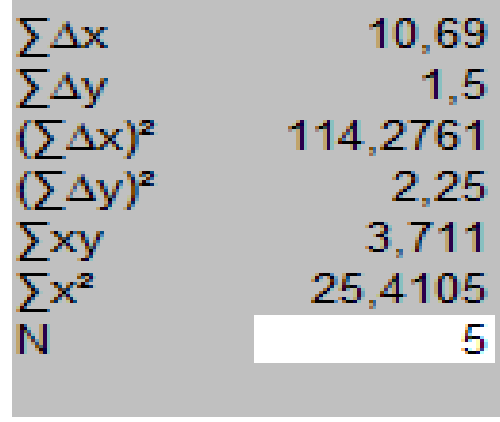

Fonte: Planilha do Excel

$\mathrm{O}$ número $\mathrm{N}$ representa o número de medidas efetuadas durante o experimento, ou seja, mostra que medimos a resistência para cinco comprimentos diferentes do fio variando de dez em dez centímetros. Com esses valores, podemos encontrar o valor de $\mathrm{D}$ e conseguinte os valores dos coeficientes angular (a) e linear (b) da reta para compor o gráfico (Figura 5).

Figura 5. Valores obtidos para D, a e b.

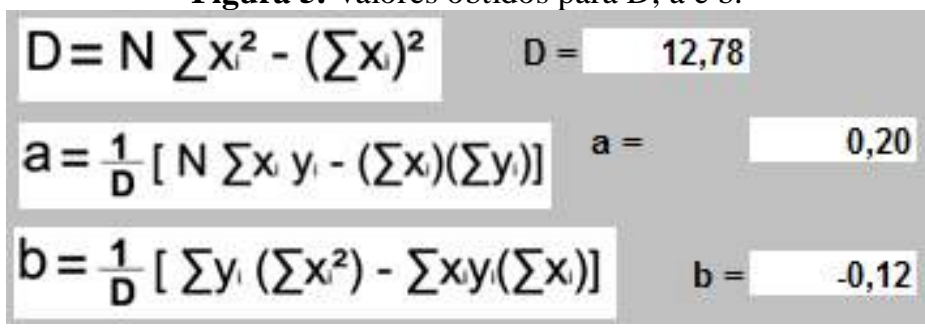

Fonte: Planilha do Excel 
(SILVA et al., 2016)

Assim, teremos agora a equação da reta e o gráfico (Figura 6) que representa o comportamento da resistência em função do comprimento do fio construído com os valores da Tabela de valores da planilha do Excel constante na Figura 3.

Figura 6. Gráfico e equação da reta.

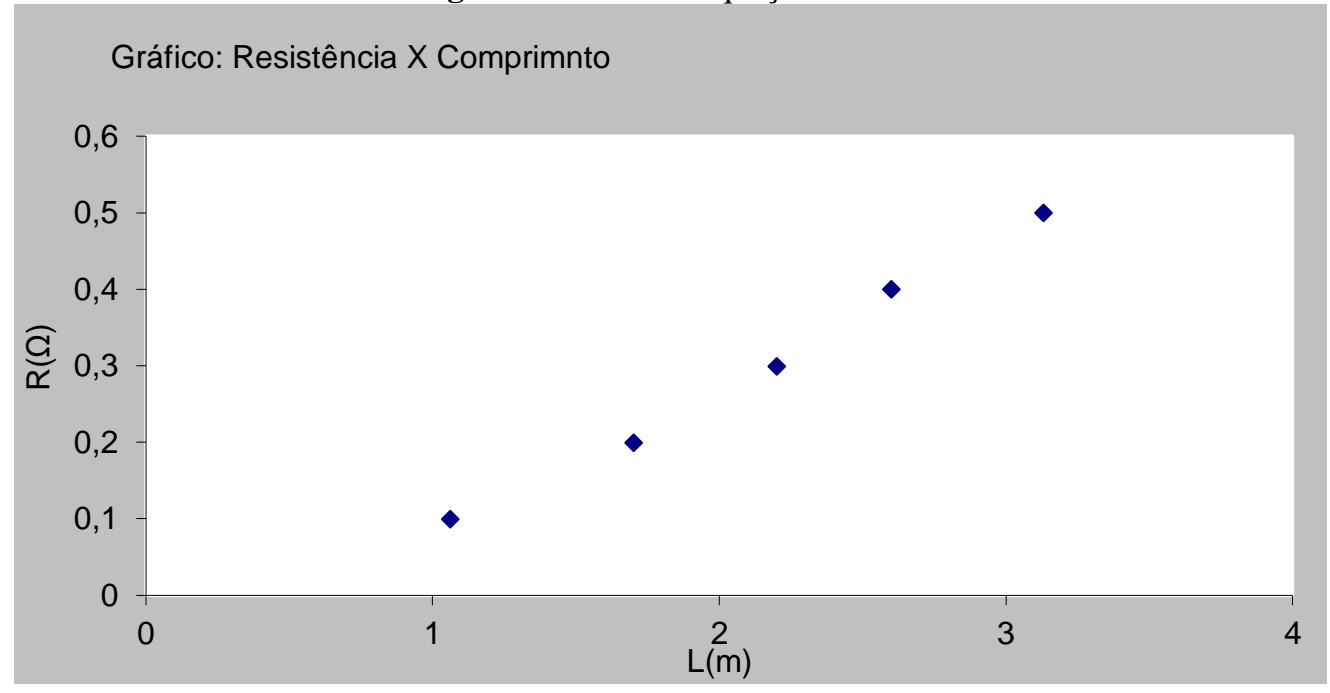

Fonte: Planilha do Excel.

A resistividade $\rho$ de um material é a resistência que este oferece ao fluxo de corrente elétrica sendo essa propriedade característica de cada tipo de material.

Foi possível neste momento da pesquisa, realiza junto com os alunos, uma análise comparativa da equação encontrada através da planilha através do método dos mínimos quadrados (Equação 2) com a expressão matemática da lei de Ohm relacionada a resistividade elétrica do material (Equação 1), conforme descrito abaixo.

Equação da lei de Ohm:

$$
R=\rho \frac{L}{A}
$$

Essa equação nos mostra que a resistência de um fio ou de outro condutor com seção reta uniforme é diretamente proporcional ao comprimento do fio e inversamente proporcional à área de sua seção reta. Ela também é proporcional a resistividade do material com o qual o condutor foi feito (SEARS et. al., 2009).
Equação obtida com a planilha do Excel:

$$
y=0,20 x-0,12
$$

Percebemos que o valor 0,20 que corresponde ao coeficiente a da equação será um valor constante que corresponde analogamente a $\rho / A$, pois a área do fio é também constante, assim como a constante de resistividade.

$O$ coeficiente $b$ representa $o$ erro experimental das medidas, este que se origina devido a fatores que interferiram no experimento. Para esse coeficiente linear o valor esperado é zero, portanto há um erro associado da ordem de $10 \%$.

Pela análise do questionário inferido após a aplicação do recurso computacional na turma de ensino médio, nota-se que o método dos mínimos quadrados aplicado à planilha contribuiu consideravelmente para o manuseio e análise dos resultados obtidos no experimento, de forma que, nas justificativas das respostas do questionário, foram apontados pontos essenciais dessa contribuição. A praticidade e a facilidade em visualizar e interpretar os resultados obtidos na prática experimental é lembrado pela maior parte dos alunos, isso levando em consideração que eles não fizeram os cálculos manualmente, 
e, dentre as respostas, pode-se destacar uma: "Sim, porque trouxe mais rapidez e praticidade na interpretação dos dados experimentais" (Aluno I).

Também foi citado o caráter da modelagem do fenômeno através da regressão linear e com isso a construção do gráfico, aspecto tal que contribui consideravelmente por sistematizar a apresentação dos dados coletados e facilitar na sua interpretação, possibilitando também uma comparação mais adequada com a teoria associada ao tema do experimento, como argumenta um Aluno II: "Sim, por apresentar toda uma sistemática para a apresentação dos resultados obtidos a partir dos experimentos realizados".

\section{Conclusões}

Buscou-se envolver os alunos do curso médio integrado em informática em atividades inovadores, para que com isso eles sejam multiplicadores do estudo feito no nosso projeto de pesquisa. Dessa forma, a ferramenta possibilitou uma compreensão mais aprofundada dos resultados experimentais, bem como da teoria associada.

Após a aplicação do método dos mínimos quadrados aliado ao experimento espera-se fazer com que os alunos do ensino médio sejam motivados e incentivados através da experimentação dentro das aulas de Física, aliando prática e teoria, de forma que os mesmos se sintam instigados pela disciplina. Também se tem a expectativa de que com uma nova forma de ensino-aprendizagem, associada à utilização de uma planilha eletrônica possa se demonstrar a aplicação e o aproveitamento do avanço tecnológico na disciplina de Física Experimental para o ensino em todos os níveis.

\section{Referências}

BETZ, M. E. M. e RIBEIRO-TEIXEIRA, R. M. Material instrucional apresentando conteúdos de métodos computacionais para o ensino de Física. Caderno brasileiro de Ensino de
Física. Florianópolis. Vol. 29, p. 787-811, 2012.

BRASIL, PCN+ Ensino Médio. Orientações Educacionais Complementares aos Parâmetros Curriculares Nacionais: Ciências da Natureza, Matemática e suas tecnologias (MEC-SEMTEC, Brasília, 2002).

CARVALHO, A.M.P.; GIL-PÉREZ, D. Formação de professores de Ciências. $4^{\mathrm{a}}$ Edição. São Paulo: Cortez, 2000.

JIMÉNEZ, M. e OTERO, L. La ciencia como construcción social. Cuardenos de Pedagogía, Barcelona, v.43, n. 180, 1990.

MEES, A. A. Implicações das teorias de aprendizagem para 0 ensino de Física. Universidade Federal do Rio Grande do SulUFRGS. Rio Grande do Sul, 2002. Disponível: <http://www.if.ufrgs.br/ amees/teorias.htm.

Acesso em 25 de junho de 2016.

MORGADO, M. J. L. Formação de professores de matemática para o uso pedagógico de planilhas eletrônicas de cálculo: análise de um curso a distância via Internet, Tese de Doutorado. UNIVERSIDADE FEDERAL DE SÃO CARLOS, 2003.

SEARS, F. et. al. FÍSICA 3 ELETROMAGNETISMO. Editora Addison Wesley, 12 $2^{\mathrm{a}}$ Edição, 2009.

SOLBES, J. e VILCHES, A. Interacciones $\mathrm{C} / \mathrm{T} / \mathrm{S}$ : un instrumento de cambio actitudinal. Revista Enseñanza de las Ciencias, Barcelona, v. 7, n. 1, 1989. 